Accretion Phenomena and Related Outflows, IAU Colloquium 163

ASP Conference Series, Vol. 121, 1997

D.T. Wickramasinghe, L. Ferrario, and G.V. Bicknell, eds.

\title{
Gaseous Disks in the Nuclei of Elliptical Galaxies
}

\author{
H. Ford, Z. Tsvetanov, L. Ferrarese, and G. Kriss
}

The Johns Hopkins University, Baltimore, MD 21218

W. Jaffe

The University of Leiden, Neils Bohr Weg. 2, Leiden, Netherlands

R. Harms and L. Dressel

RJH Scientific Inc., Alexandria, VA 22303

\begin{abstract}
HST images have led to the discovery that small $\left(r \sim 1^{\prime \prime}\right.$; $r \sim 100-200 \mathrm{pc}$ ), well-defined, gaseous disks are common in the nuclei of elliptical galaxies. Measurements of rotational velocities in the disks provide a means to measure the central mass and search for massive black holes in the parent galaxies. The minor axes of these disks are closely aligned with the directions of the large-scale radio jets, suggesting that it is angular momentum of the disk rather than that of the black hole that determines the direction of the radio jets. Because the disks are directly observable, we can study the disks themselves, and investigate important questions which cannot be directly addressed with observations of the smaller and unresolved central accretion disks. In this paper we summarize what has been learned to date in this rapidly unfolding new field.
\end{abstract}

\section{Introduction}

Astronomers have long speculated that quasars and active galactic nuclei (AGN) are powered by massive black holes which are fed by accretion disks. There is direct evidence that the central engines of Seyfert 2 galaxies are surrounded by dusty tori which hide the engines from direct view (Antonucci and Miller, 1985). The widely accepted AGN paradigm states that all Seyfert 1 and Seyfert 2 galaxies have a cold, dusty molecular torus which surrounds the central hot accretion disk and massive black hole. The two types of Seyferts then differ in appearance depending on whether or not the orientation of the torus hides the accretion disk and associated broad emission line region from direct view. The most interesting postulate of the AGN paradigm, the presence of a massive black hole, has been confirmed by VLBA measurements of a $3.5 \times 10^{7} \mathrm{M}_{\odot}$ black hole in NGC 4258 (Miyoshi et al. 1995), and Hubble Space Telescope (HST) measurements of $2 \times 10^{9} \mathrm{M}_{\odot}, 5 \times 10^{8} \mathrm{M}_{\odot}$, and $2 \times 10^{9} \mathrm{M}_{\odot}$ black holes in M87 (Harms et al. 1994, Ford et al. 1996), NGC 4261 (Ferrarese et al. 1996), and NGC 3115 (Kormendy et al. 1996). And, as we discuss below, HST has shown 
that there is direct evidence for dusty disks in AGNs, albeit with sizes larger than expected.

An HST broad-band survey of a complete sample of elliptical galaxies in the Virgo cluster by Jaffe et al. $(1993,1994)$ revealed a sharp-edged, dusty disk with radius $r \sim 120 \mathrm{pc}$ in the center of NGC 4261 . The fact that the axis of the large scale radio jets is within $13^{\circ}$ of the disk's projected minor axis strongly suggests that the direction of the radio jet is aligned by the dusty disk. After finding the disk in NGC 4261, we searched for a disk in M87 immediately after the first HST servicing mission and found a disk of ionized gas whose minor axis is within $10^{\circ}-20^{\circ}$ of the axis the synchrotron jet (Ford et al. 1994). Subsequent HST Faint Object Spectrograph measurements of the radial velocities in these disks showed that the disks are in approximate Keplerian motion around massive black holes( Harms et al. 1984).

In this paper we show that small $\left(r \sim 1^{\prime \prime}\right)$, well-defined disks are common in elliptical galaxies. These previously unobserved disks, which we refer to as nuclear disks, are very interesting. Measurements of rotational velocities in the disks provide a means to determine the central mass in the parent galaxy. Because the disks are directly observable, we can study the disks themselves (e.g. NGC 4261, Jaffe and McNamara, 1994, Ferrarese et al. 1996, and M87, Tsvetanov et al. 1997, 1997b, Dopita 1997), and investigate important questions which cannot be directly addressed with observations of the smaller and unresolved central accretion disks. We can measure the variation of turbulence across the disks, observe warps and spiral arms in the disks, determine how the disks are ionized, and in principle establish whether or not there are magnetic fields in the disks.

\section{Surveys of Early Type Galaxies}

We will concentrate on the published results from two WFPC1 surveys, and the results from a continuing WFPC2 survey by the authors and colleagues. Jaffe et al. (1994: J94) selected a sample of elliptical galaxies unbiased by any criteria other than membership in the Virgo cluster as defined by Huchra (1984), classification as E or E/S0 in the RSA (Sandage and Tammann 1981), and brighter than $B_{T}=13.45$. This statistically complete, luminosity-limited sample consists of the 12 brightest $\mathrm{E}$ and $\mathrm{E} / \mathrm{S} 0$ galaxies in the Virgo cluster, plus M87 and NGC 4472, which were added to the sample from the HST archives. Because the Virgo cluster is relatively near $(D \sim 15-17 \mathrm{Mpc})$, the galaxies could be observed and compared at the same high spatial resolution without differences and uncertainties introduced by different distances.

The frequency of dust and disks or disk-like structures found by J94 is summarized in Table 1. The third column gives the photometric type defined by $\mathrm{J} 94$, the fourth column lists whether the disk is primarily ionized as in M87, dusty as in NGC 4261, or a mixture of neutral and ionized gas, the fifth column gives the apparent radius of the disk in parsecs, and the last column gives the angle between the minor axis of the disk and the radio and/or optical jet. Images and photometric profiles of these galaxies and disks can be found in J94, van den Bosch et al. 1994, and Ferrarese et al. 1994. 
TABLE 1

Gaseous Disks in the 14 Brightest Virgo E and E/SO Galaxies

\begin{tabular}{lcclcc}
\hline Galaxy & $\begin{array}{c}\text { RSA } \\
\text { Type }\end{array}$ & $\begin{array}{c}\text { Photometric } \\
\text { Type }\end{array}$ & \multicolumn{1}{c}{ Type of Disk } & $\begin{array}{c}\text { Radius } \\
(\mathrm{pc})^{*}\end{array}$ & $\begin{array}{c}\text { Radio to Minor } \\
\text { Axis Angle }\end{array}$ \\
\hline 4486 & EO & I & HII/(Dusty) & $\sim 80$ & $10-20$ \\
4261 & E3 & I & Dusty/HII & $\sim 120^{* *}$ & $\sim 13$ \\
4374 & E1 & I & Dusty/HII Disk-like & $\sim 400$ & $\sim 0$ \\
4476 & E5pec & II & Dusty Disk-like & $\sim 500$ pc & \\
\hline
\end{tabular}

*(at $17 \mathrm{Mpc})$

*At an assumed distance of $30 \mathrm{Mpc}$

The dust in NGC 4374 (cf. J94) has a sinusoidal, striated, disk-like structure that is very reminiscent of the dust lanes in NGC 5128 (Cen A), which are known to be a large disk of captured dust and gas which is settling into one of the principle planes of Cen A's triaxial potential. We think that much the same thing is happening in NGC 4374. The dusty disk-like structure in NGC 4476 has an obvious spiral structure. The alignment between the orientation of the disks and disk-like structure and the radio jets in the three radio galaxies in Table 1 suggests a causal relationship between the angular momentum in the dust and the direction of the radio jets.

Lauer et al. (1995: L95) observed a heterogeneous sample of 45 galaxies with a selection emphasis designed to fill out the core parameter relations of Lauer (1985) and Kormendy (1985). The sample includes Local Group Es, high luminosity galaxies that appeared to have small cores, galaxies like NGC 3115 that are candidates to host MBHs, four M32-like dwarfs in Virgo, and "a few galaxies with unusual central morphologies and kinematics". Excluding Abell $\mathrm{cD}$ galaxies and M32-type dwarfs, there are $34 \mathrm{E}$ and $\mathrm{E} / \mathrm{S} 0$ galaxies in the L95 sample. Three of the ellipticals have dusty nuclear disks, and one S0 has a disklike structure. Table 2 lists the galaxies with disks and gives the RSA types, the $\mathrm{J} 94$ photometric type, the approximate radius of the disk in parsecs, and whether or not the galaxy has ionized gas in the nucleus as determined from $\mathrm{H} \alpha$ spectra taken by one of the authors (HCF). Figure 1 is an image of the beautiful NGC 4261-type disk in NGC 4697, taken from L95, and, for comparison, the disk in NGC 4261.

The J94 and L95 surveys show that dust and nuclear disks are common in elliptical galaxies. J94 found detectable dust in $\sim 70 \%$ of the Virgo ellipticals, and found disks or disk-like structures in $\sim 30 \%$ of the galaxies in their complete sample. Although the L95 sample was biased against dust, they found dust in $\sim 30 \%$ of their galaxies, and disks or disk-like structures in $\sim 10 \%$ of their galaxies.

Van Dokkum and Franx (1995: vDK95) studied the dust in (all) 64 Cycle 1 through 3 HST Archival WFPC1 images of early-type galaxies. They found that dust is detected in $\sim 72 \% \pm 16 \%$ of radio-loud ellipticals and in $\sim 33 \% \pm 9 \%$ of radio quiet ellipticals. After correction for bias against detection of face-on disks (because of small optical depth), they conclude that the true frequency of 
TABLE 2

Gaseous Nuclear Disks in Faber and Lauer E and E/SO Galaxies

\begin{tabular}{lcclcc}
\hline \hline Galaxy & $\begin{array}{c}\text { RSA } \\
\text { Type }\end{array}$ & Type & $\begin{array}{c}\text { Disk } \\
\text { Disk-like }\end{array}$ & $\begin{array}{c}\text { Radius } \\
(\mathrm{pc})^{*}\end{array}$ & Nuclear HII \\
\hline 4697 & E6 & II & Disk & $\sim 175$ & Y \\
5322 & E/3 & $?$ & Disk & $\sim 130^{* *}$ & Y \\
5845 & E3 & II & Disk & $\sim 100$ & N \\
4150 & S0 & $?$ & Disk-like & $\sim 30 \mathrm{pc}$ & Y \\
\hline
\end{tabular}

${ }^{*}$ Using Lauer et al. (1994) distances
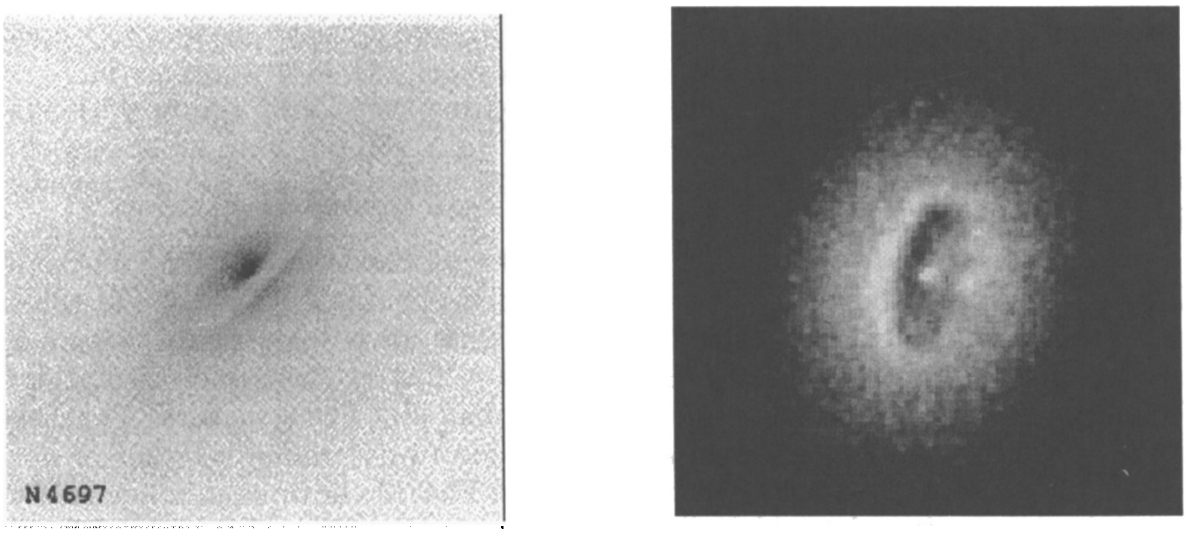

Figure 1. The left panel shows the dusty disk in NGC 4697. The disk has a similar size $(r \sim 175 \mathrm{pc})$ and appearance as the disk in NGC $4261(r \sim 120$ pc) shown on the right. Both disks have sharp edges. 

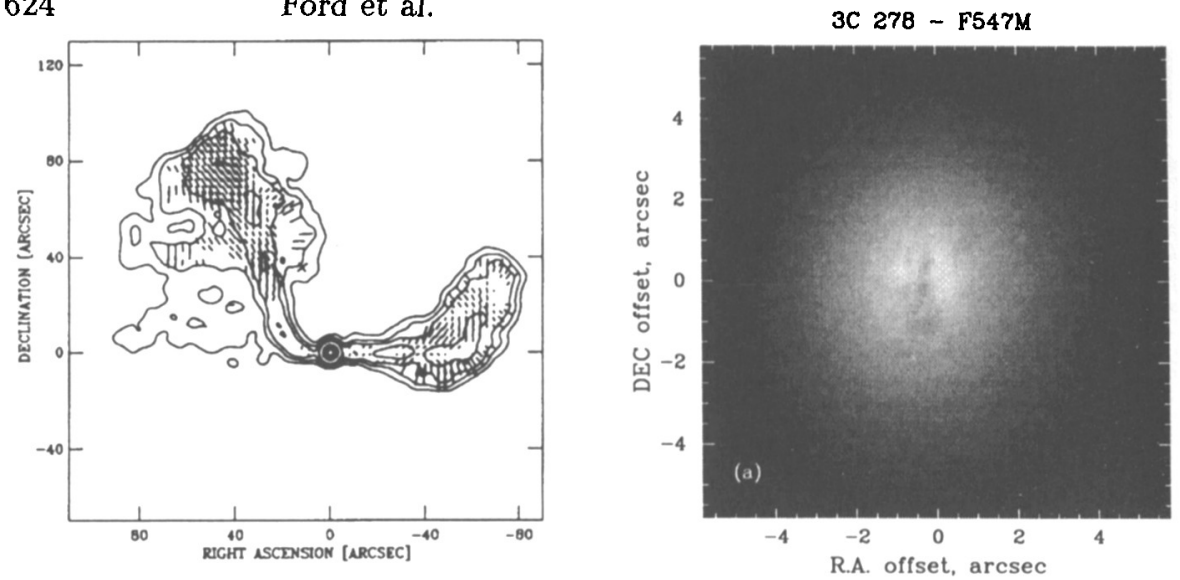

Figure 2. The left panel shows the large-scale radio map of 3C278 and the right hand panel shows the small $(r \sim 300 \mathrm{pc})$, irregular, dusty disk in 3C278. The disk is nearly edge-on and is perpendicular to the radio jets. The radio jet bends on one side because of the presence of a nearby galaxy which is either a companion or a close encounter with $3 \mathrm{C} 278$.

dust in ellipticals is $\sim 78 \% \pm 16 \%$. After comparing the vDK95 results with J94 and L95, it is clear that large scale dust lanes $(a>250 \mathrm{pc})$ have an elongated, irregular appearance, whereas small-scale dusty disks $(a \leq 200 \mathrm{pc})$ are regular in appearance and often have sharp edges.

We presently are using HST WFPC2 $\mathrm{H} \alpha$ on-band and F547M off-band images to search for nuclear disks in radio-loud ellipticals. Two of the three radio galaxies imaged to date, $3 \mathrm{C} 278$ and $3 \mathrm{C} 264$, have dusty disks. Figure 2 shows a radio map and the $\mathrm{F} 547 \mathrm{M}$ image of $3 \mathrm{C} 278$ at the same orientation. The inner radio jets are perpendicular to the small disk-like structure. The disk in $3 \mathrm{C} 264$ is nearly face-on, making it impossible to determine whether or not the radio jet is perpendicular to the disk. The F547M continuum image shows a bright synchrotron jet which disappears in the $\mathrm{H} \alpha$ on-band minus off-band image. The latter image shows a small, circular disk of ionized gas. If the synchrotron and radio jet are nearly perpendicular to the disk as we think is the case in other radio galaxies with nuclear disks, the jet is highly beamed.

\section{Conclusions and Discussion}

HST images show that dust and dusty nuclear disks are common in early type galaxies. The minor axes of these disks are closely aligned with the directions of the large-scale radio jets. If the disks are the result of stochastic accretion events, the angular momentum of the central black hole will not necessarily be aligned with that of the large-scale disk. Consequently, the preferential alignment of radio jets with the disk normals suggests that it is angular momentum of the disk rather than that of the black hole that determines the direction of the radio jet. 
These observations raise many interesting questions about the nature of the nuclear disks and their interaction with a central massive black hole. Why do the disks have sharp edges? What does this tell us about the central potential? How is angular momentum transported in the disks? Are magnetic fields important? How is the gas ionized and excited? What is the disk structure (warps, eccentricity, spiral arms, etc.), and how do we account for it? What are the kinematics in the disks? How do we account for the turbulence?

The discovery and study of a large number of nuclear disks will eventually lead to answers to these questions.

Acknowledgments. This research was supported by NASA grants NAS 5-29293, NAG5-1630, and GO-05432.01-93A.

\section{References}

Antonucci, R.R.J. and Miller, J.S., 1985, ApJ, 297, 621

Dopita, M., 1997, preprint

Ferrarese, et al., 1994, AJ, 108, 1598

Ferrarese, L., Ford, H.C., and Jaffe, W., 1996, ApJ (in press)

Ford, H.C., et al., 1994, ApJ, 435, L27

Ford, H.C., et al., 1996, in Science with the Hubble Space Telescope - II, ed. P. Benvenuti, F.D. Macchetto, E.J. Schreier (Baltimore, MD: STScI), 192

Harms, R.J., et al., 1994, ApJ, 435, L35

Huchra, J.P. (1984): in The Virgo Clusters of Galaxies, (ESO Conference Proc.

2), ed. O.-G. Richert and B. Binggeli, p.181

Jaffe, W., et al., 1993, Nature, 364, 213

Jaffe, W. et al., 1994, AJ, 108, 1567

Jaffe, W., McNamara, B.R., 1994, ApJ, 434, 110

Kormendy, J., 1985, ApJ, 295, 73

Kormendy, J., et al., 1996, ApJ, 459, L57

Lauer, T.R., 1985, ApJ, 292, 104

Lauer, T.R., et al., 1995, AJ, 110, 2622

Miyoshi, M. et al. 1995, Nature, 373 , 127

Sandage, A., and Tammann, G.A. 1981, A Revised Shapley-Ames Catalogue of Bright Galaxies (Washington, DC: Carnegie Institute of Washington)

Tsvetanov, Z., et al., 1997, in preparation

van den Bosch, F.C.,et al., 1994, AJ, 108, 1579

van Dokkum, P.G., \& Franx, M., 1995, AJ, 110, 2027

\section{Discussion}

\section{G. Bicknell: What are the thicknesses of these disks?}

H. Ford: The disk in NGC 4261 is less than 0.1 " at the edge, based on projection of a "poker chip" onto the sky. At the estimated distance of $30 \mathrm{Mpc}$, this corresponds to a thickness $\leq 15$ pc. 